\title{
A Quick Tables Look-up Optimization Algorithm for CAVLC Decoding
}

\author{
Jianhua Wang ${ }^{1}$, Lianglun Cheng ${ }^{1}$, Jun Liu ${ }^{1}$ and Tao Wang ${ }^{1}$ \\ ${ }^{1}$ Faculty of Automation, Guangdong University of Technology, Guangzhou, China \\ 123chihua@163.com, llcheng@gdut.edu.cn, liujun7700@163.com' \\ 466586789@qq.com
}

\begin{abstract}
In this paper, a quick table look-up optimization algorithm is presented to solve the problems of long table look-up time for CAVLC decoding in H.264/AVC. The achievement of the new algorithm rests that we make full use of the hash table query and index technology to improve the table look-up speed for CAVLC decoding. The basic idea of the new algorithm is that we take the number of zero in code prefix calculated from input bit-stream as index key of the first level, the value of codeword suffix as index key of the second level, then through index key of the one and second index key above, we can quickly get the decoded codeword located in the third level in a hash table built, which can reduce a lot of table look-up time for CAVLC decoding in H.264/AVC. The simulation results show that our proposed schemes based on hash-index method can reduce about $40 \%$ table look up time for CAVLC decoding compared with TLSS method, without degrading video quality.
\end{abstract}

Keywords: CAVLC decoding, Table look-up, Hash-index query

\section{Introduction}

H.264/AVC is the latest international video coding standard, which has been developed by ITU-T and ISO/IEC [1]. It has greatly improved the compression ratio and video quality and has been widely used in video communication aspects. H.264/AVC has three kinds of levels: Baseline profiles, Main profiles and extended profiles. Baseline profiles are mainly used in a wide range of small size equipments; Main profiles are adopted to improve image quality and compression ratio; extended profiles are applied to networking video streaming transmission.

In the Baseline profiles of H.264/AVC, Context-based Adaptive Variable Length Coding (CAVLC) as an entropy coding tool is used to decode residual blocks. It increases the compression ratio and video quality, at the same time, it also increases power consumption and hardware cost of the decoder due to frequent table look up. During the process of CAVLC decoding, there are five syntax elements to decode, and three in five syntax elements, Coeff_token, Run_before and Total_zeros, need to use variable length tables to decode code, while the rest of them, Level and sign of TrailingOnes (T1s), are decoded by the regular arithmetic operations without using variable length tables look-up. So Tables look-up problem is a very important problem for CAVLC decoding in H.264/AVC. It have been reported that looking-up variable length tables occupy about $96.6 \%$ time of the CAVLC decoding in CAVLC decoding and Tables look-up time have became one important performance and power bottleneck in embedded systems especially for multimedia applications [2]. In this paper, tables look up time refers variable length tables look up time. 
In order to reduce table look-up time for CAVLC decoding, previous many researchers have proposed various solutions for it. Some general Table Look-up methods, such as Table Look-up by Sequential Search (TLSS), Table Look-up by Binary Search (TLBS), are proposed to improve table look-up speed. Heng, et al., [3] merged all codeword tables into one table and organized the table into sub-tables to save table look-up time, reducing $40 \%$ power consumption. Lee, et al., [4] proposed pipelined architecture to save the operation frequency greatly, saving table look-up time. Wang, et al., [5] presented a novel low-cost high-performance CAVLC decoder for H.264/AVC which greatly improved CAVLC decoding speed. Huang, et al., [6] proposed a decoder based on CMOS and FPGA technology which reduces power consumption by $44-48 \%$ more than previous low-power CAVLD schemes. Moon, et al., [7] proposed a new VLDs based on integer arithmetic operations for Run_before and Total_zeros to reduce some table look-up time and reduce memory access [7]. Lu, et al., [8] proposed one kind of entropy decode algorithm, which can decrease great amount of time than the original algorithm in the H.264 reference software; Lee, et al., [9] developed new codeword structures, look-up tables and searching methods for the CAVLC syntax elements to reduce the table look up time. Kim, et al., [10] proposed some other integer arithmetic operations to improve greatly table look-up speed. To reduce the time of table matching, a fast method was proposed by using Table Grouping to construct custom CAVLC decoding tables in this paper [11].

In this paper, we propose a quick table look-up optimization algorithm based on hashindex query for CAVLC decoding. Our algorithm uses hash table and index technology to improve the performance of table look up for CAVLC decoding, which can reduce a lot of tables look up time by decreasing codes looking-up and judging operations. The simulation results show that our proposed scheme can reduce $40 \%$ table look-up time compared with TLSS method for CAVLC decoding without degrading video quality.

The rest of this paper is organized as follows. In Section 2, the principle and common optimization method of CAVLC decoding is introduced. The proposed decoding method based on hash-index query is presented in Section 3. The simulation result of proposed scheme compared with general methods is presented in Section 4. In Section 5, we give some conclusions.

\section{The Principle and Common Optimization Method of CAVLC Decoding}

\subsection{Principle of CAVLC Decoding}

In baseline profile of H.264/AVC, CAVLC, as one entropy decoding method, is adopted to decode the quantized transform coefficients for residual blocks. The CAVLC decoding decodes residual data into five important syntax elements [9]. These five syntax elements are Coeff_token, Sign of TrailingOnes, Level, Total_zeros and Run_before respectively. Figure 1 is the decoding order and the definition of five syntax elements above are described as follows.

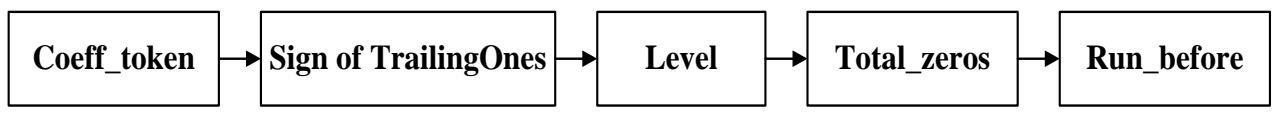

Figure 1. Decoding Order of Five Syntax Elements

Coeff_token: Both the number of nonzero coefficients (Totalcoeff) and number of coefficients that absolute value is equal to one (TrailingOnes). It is decoded by a FixedLength Code Tables and three variable length Tables. 
- Sign of TrailingOnes (T1s): Use a single sign bit, which 0 is for positive and 1 is for negative, to represent each T1s in reverse zigzag order. It is decoded by maximum 3-bit reading without any table look-up.

- Level: The values for each nonzero coefficient except for T1s in reverse zigzag order. It is decoded in reverse order and the output of this process is a list of different Level

- Total_zeros: The total number of zero coefficients between the DC and the last nonzero coefficient in zigzag order. It is decoded by looking up some corresponding variable length Tables.

- Run_before: The numbers of zeros preceding each nonzero coefficient in reverse zigzag order. It is decoded by looking up some corresponding variable length tables.

\subsection{Common Optimization Method of CAVLC Decoding}

Table look-up is a very time-consumption operation in most equipments. As the process of CAVLC decoding involves many table look up operation, it seriously affect the efficiencies of CAVLC decoding. In order to improve efficiency of CAVLC decoding, recently some efficient methods have been adopted to improve table look-up methods. According to analyzing the methods used in at present, they can be summarized into the following classifications.

1) Table Lookup by Sequential Search (TLSS) method: This method is used to look up table by comparing one bit at a time with the decoding table. As it usually needs to research whole code tables to find the desired codeword, this method requires multiple table look-up time.

2) Table Look-up by Binary Search (TLBS) method: This method mainly make full use of advantages of binary tree's high search efficiency and the sub-table's simple storage structure to build a binary tree for each sub-table. This algorithm could greatly improve the search efficiency for code table. Disadvantages of this method lie in its large storage space.

3) Table Look-up by Sub-table (TLST) method: the basic idea of this method is that it mainly changes the original two-dimension table into many small parts. As this method needs to judges key words to determine the node in which block to be searched and to make sequential search or half search in certain pieces, it requires prolonged running time. This method is not suitable for real-time processing.

4) Table Look-up by Code Grouping (TLTG) method: This method gets the number of TC and T1s through calculation based on the number of 0 before 1 in the code stream. As this method cannot get the length of the stream immediately, its defects rests that it needs long time to feedback the length of the code stream.

5) Table Look-up by arithmetic operations (TLAO) method: This method mainly use to arithmetic operations look-up instead of the conventional table look-up method. It can achieve a fast decoding and reduced power consumption by decreasing memory accesses.

\section{Proposed Scheme}

\subsection{Observe Variable Length Tables}

As mentioned above, the process of CAVLC decoding needs to decode five syntax elements. Three in five syntax elements, Coeff_token, Run_before and Total_zeros, need to look up variable length Tables. Through analysis the variable length Tables, we can find that Coeff_token has three 2D-variable length tables: $0<=\mathrm{NC}<2,2<=\mathrm{NC}<4,4<=\mathrm{NC}<8$; while the Run_before and Total_zeros VLDs have one 1D-variable length Tables. Table 1 is the part 
codewords of 2D-variable length Tables for Coeff_token $(0 \leq \mathrm{NC}<2)$. Table 2 is the part codewords of 1D-variable length Tables for Total_zeros $(\mathrm{Tc}=5)$. In this paper, we mainly need to optimize the Table look-up algorithm for those variable length Tables above.

Table 1. Part of 2D-Variable Length Table for Coeff_token $(0 \leq N C<2)$

\begin{tabular}{|c|c|c|}
\hline Code & Codeword & {$[\mathrm{T} 1, \mathrm{Tc}]$} \\
\hline 1 & $0 \times 00$ & {$[0,0]$} \\
\hline 01 & $0 \times 21$ & {$[1,1]$} \\
\hline 001 & $0 \times 42$ & {$[2,2]$} \\
\hline 000100 & $0 \times 22$ & {$[1,2]$} \\
\hline 000101 & $0 \times 01$ & {$[0,1]$} \\
\hline 00011 & $0 \times 63$ & {$[3,3]$} \\
\hline 000011 & $0 \times 64$ & {$[3,4]$} \\
\hline 0000100 & $0 \times 65$ & {$[3,5]$} \\
\hline 0000101 & $0 \times 43$ & {$[2,3]$} \\
\hline$\ldots$ & $\ldots \ldots$ & $\ldots \ldots$ \\
\hline 000000000000001 & $0 \times 2 d$ & {$[1,13]$} \\
\hline
\end{tabular}

Table 2. Part of 1D-variable Length Table for Total_zeros (Tc=5)

\begin{tabular}{|c|c|}
\hline Code & Codeword \\
\hline 111 & 3 \\
\hline 110 & 4 \\
\hline 101 & 5 \\
\hline 100 & 6 \\
\hline 0101 & 0 \\
\hline 0100 & 1 \\
\hline 011 & 7 \\
\hline 0011 & 2 \\
\hline 0010 & 8 \\
\hline 0001 & 10 \\
\hline 00001 & 9 \\
\hline 00000 & 11 \\
\hline
\end{tabular}

In Table 1, the code represents the input bit-stream of the Coeff_token syntax element $(0 \leq \mathrm{NC}<2)$. The 8-bit codeword represents the decoded output and the front 3 bits of them are total number of ones (T1) and the other tail 5 bits are the total number of coefficients (Tc). In Table 2, the code stands for the input bit-stream of the syntax elemen of Total_zeros, the codeword stands for single decoded output directly $(\mathrm{T} c=5)$. Note that the other elements of Coeff_token, Run_before and Total_zeros have the same code Table structure as Table 1 and Table 2.

\subsection{Find Internal Relationship between Code Length and Numbers of 0 in Code Prefix}

By analyzing the codeword structure in Table 1 and Table 2 above, we find that there are some internal relationships between numbers of 0 in code prefix and code length for Coeff_token and Total_zeros, which are shown as in Table 3 and Table 4 respectively. 
Table 3. The Relationship Exists between Code Length and Numbers of 0 in Code Prefix Corresponding to Table 1

\begin{tabular}{|c|c|c|c|c|c|c|c|c|c|c|c|c|c|c|c|}
\hline $\begin{array}{c}\text { Numbers of } 0 \\
\text { in code prefix }\end{array}$ & 0 & 1 & 2 & 3 & 4 & 5 & 6 & 7 & 8 & 9 & 10 & 11 & 12 & 13 & 14 \\
\hline Code length & 1 & 2 & 3 & 5or 6 & 6 or 7 & 8 & 9 & 10 & 11 & 13 & 14 & 15 & 16 & 16 & 15 \\
\hline
\end{tabular}

Table 4. The Relationship Exists between Length of Code and Numbers of 0 in Code Prefix Corresponding to Table 2

\begin{tabular}{|c|c|c|c|c|c|c|}
\hline Numbers of 0 in code prefix & $\underline{0}$ & 1 & 2 & 3 & 4 & 5 \\
\hline Code length & 3 & 3 or 4 & 4 & 4 & 5 & 5 \\
\hline
\end{tabular}

Table 3 and Table 4 are the internal relationships between the length of code and numbers of 0 in code prefix for Coeff_token and Total_zeros respetively. Through the internal relationship, we can find a new Table look-up method to quickly determine the length of code suffix from input decoded bit-stream, which can save lots of time of looking up and judging code suffix.

\subsection{Build Hash-index Table}

Based on this principle above, we build hash-index table to express the relationships existed in code and numbers of 0 and length of code prefix and code length. The built hashindex tables for 2D-Coeff_token and the 1D-Total zeros are shown as Table 5 and Table 6 respectively.

Table 5. Part hash-index Table of 2D-Table for Coeff_token (VLCT0, $0 \leq \mathrm{NC}<2$ )

\begin{tabular}{|c|c|c|c|c|c|}
\hline Code & $\begin{array}{c}\text { Hash } \\
\text { address }\end{array}$ & $\begin{array}{l}\text { Numbers of } 0 \\
\text { in code prefix }\end{array}$ & $\begin{array}{c}\text { Value of } \\
\text { code suffix }\end{array}$ & $\begin{array}{l}\text { Index } \\
\text { value }\end{array}$ & $\begin{array}{l}\text { Code- } \\
\text { word }\end{array}$ \\
\hline 1 & 0 & 0 & 1 & 01 & $0 \times 00$ \\
\hline 01 & 1 & 1 & $\mathrm{x}$ & $1 \mathrm{x}$ & $0 \times 21$ \\
\hline 001 & 2 & 2 & $\mathrm{x}$ & $2 x$ & $0 \times 42$ \\
\hline 000100 & \multirow{3}{*}{3} & \multirow{3}{*}{3} & 00 & 300 & $0 \times 22$ \\
\hline 000101 & & & 01 & 301 & $0 \times 01$ \\
\hline 00011 & & & 1 & 31 & $0 \times 63$ \\
\hline 000011 & \multirow{3}{*}{4} & \multirow{3}{*}{4} & 1 & 41 & $0 \times 64$ \\
\hline 0000100 & & & 00 & 400 & $0 \times 65$ \\
\hline 0000101 & & & 01 & 401 & $0 \times 43$ \\
\hline$\ldots$ & $\ldots$ & $\ldots \ldots$ & $\ldots \ldots$ & $\ldots \ldots$ & $\ldots \ldots$ \\
\hline 000000000000001 & 14 & 14 & $\mathrm{X}$ & $14 x$ & $0 \times 2 d$ \\
\hline
\end{tabular}

Table 6. Part Hash-index Table for Total_zeros (Tc=5)

\begin{tabular}{|c|c|c|c|c|c|}
\hline Code & Hash address & $\begin{array}{l}\text { Numbers of } 0 \\
\text { in code prefix }\end{array}$ & Code suffix & Index value & Codeword \\
\hline 111 & \multirow{4}{*}{0} & \multirow{4}{*}{0} & 111 & 0111 & 3 \\
\hline 110 & & & 110 & 0110 & 4 \\
\hline 101 & & & 101 & 0101 & 5 \\
\hline 100 & & & 100 & 0100 & 6 \\
\hline 0101 & \multirow{3}{*}{1} & \multirow{3}{*}{1} & 01 & 101 & 0 \\
\hline 0100 & & & 00 & 100 & 1 \\
\hline 011 & & & 1 & 11 & 7 \\
\hline 0011 & 2 & 2 & 1 & 21 & 2 \\
\hline
\end{tabular}




\begin{tabular}{|c|c|c|c|c|c|}
\hline 0010 & & & 0 & 20 & 8 \\
\hline 0001 & 3 & 3 & $x$ & $3 \mathrm{x}$ & 10 \\
\hline 00001 & 4 & 4 & $\mathrm{x}$ & $4 \mathrm{x}$ & 9 \\
\hline 00000 & 5 & 5 & $\mathrm{z}$ & $5 \mathrm{z}$ & 11 \\
\hline
\end{tabular}

In Table 5 and Table 6, the code represents the input bit-stream of the corresponding syntax element. Hash Table addresses are allocated mainly according to numbers of 0 in code prefix of code. Numbers of 0 in code prefix of code could be mapped to hash table addresses by a hash function in hash-index Table. The length of code suffix is determined through the relation existed in code length and numbers of 0 in code prefix, such as Table 3 and Table 4. We takes number of zero in code prefix calculated from input bit-stream as index key of the first level, the value of codeword suffix as index key of the second level, and through the index key of the one and second index key above, we can quickly get the decoded output located in third level in a hash table built.

\subsection{Realize Tables Look-up Algorithm based on Hash-index Query}

Based on the ideas above, we propose a table look-up algorithm based on hash-index query for CAVLC decoding. The basic idea of new algorithm is that we takes number of zero in code prefix calculated from input bit-stream as index key of the first level, the value of codeword suffix as index key of the second level, and through the determined index value of the one and second above, we can quickly get the decoded output located in third level in a hash Table built, which can save a lot of Tables look-up time. The process of Table look-up algorithm based on hash-index query could be shown as Figure 2.

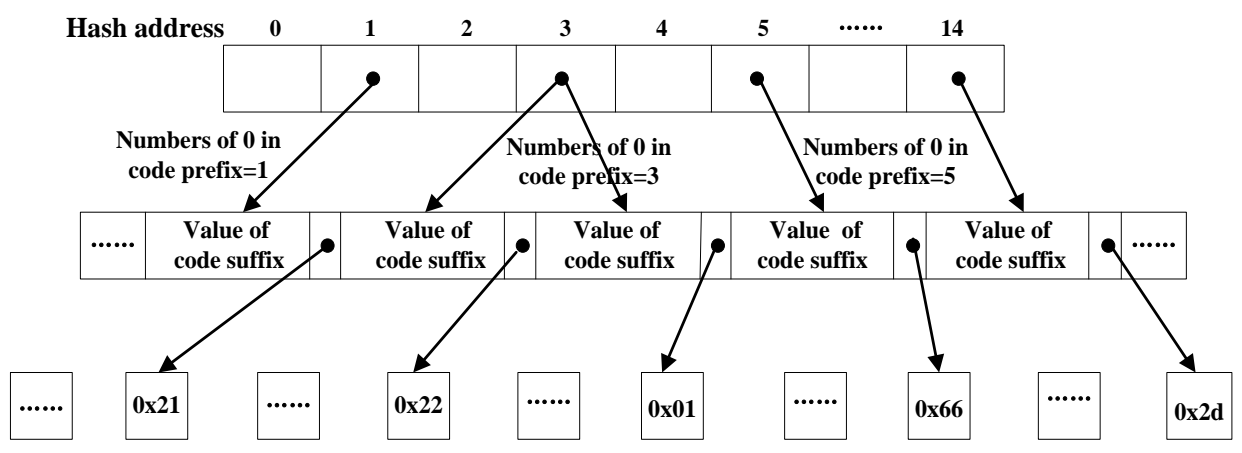

Figure 2. Table Look-Up Algorithm based on Hash-Index Query

The proposed table look-up algorithm based on hash-index query for CAVLC decoding can be summed up some steps as follows.

Step 1: select variable length tables of Coeff_token syntax element through the value of NC.

Step 2: read input decoded bit-stream and calculate numbers of 0 in code prefix as index key of the first level

Step 3: find the length of code suffix in second level by numbers of 0 in code prefix mapped in built hash table.

Step 4: determine the length of code suffix according to length of code suffix or the first value of code suffix. If the length of code suffix found has two possible values, we can get the right length of code suffix after judging the first value of code suffix again. 
Step 5: read the value of code suffix as index key of the second level according to the numbers of 0 in code prefix and the length of code suffix above.

Step 6: find decoded codeword in third level through the determined index key of the one and second above.

\section{Table 7. Is pseudo Code of Tables Look-up based on hash-index Algorithm}

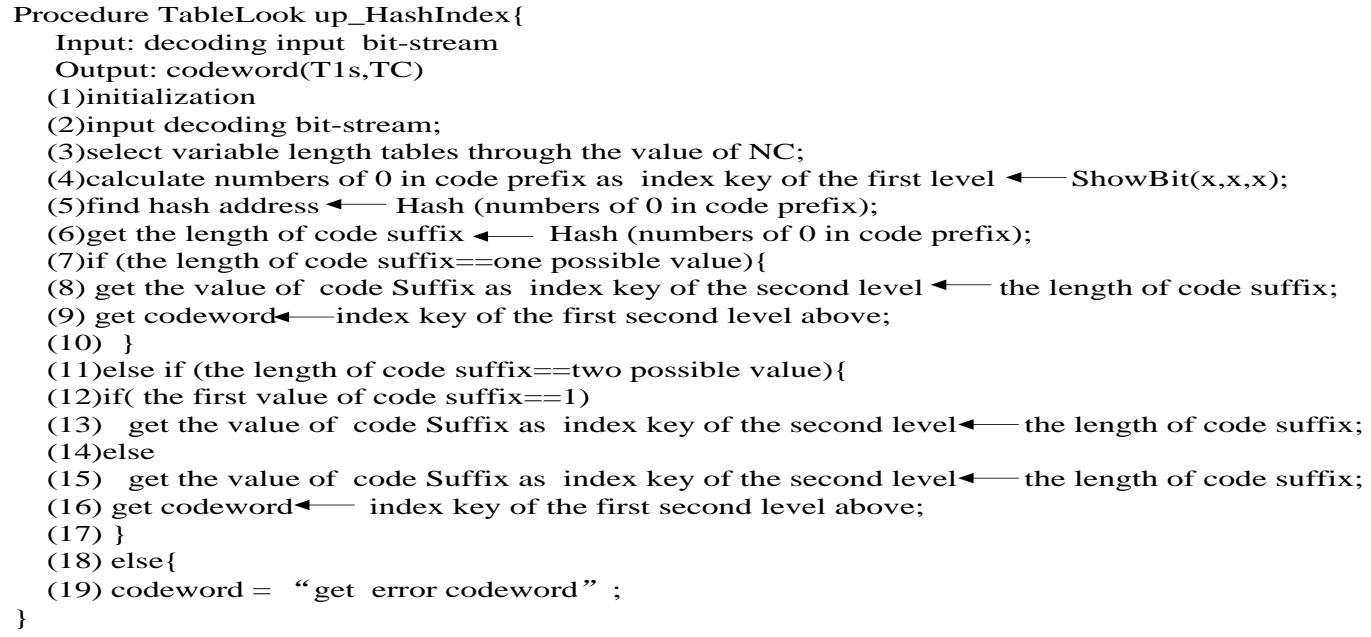

\subsection{Demonstrate Method Instance}

Take decoding Coeff_token element for an example to illustrate our proposed method based on hash-index method. And supposed that $\mathrm{NC}$ value is $0 \leq \mathrm{NC}<2$, the bit-stream inputted is $0000100011 \ldots$...Figure 3 is the decoding process of an example with our proposed scheme.

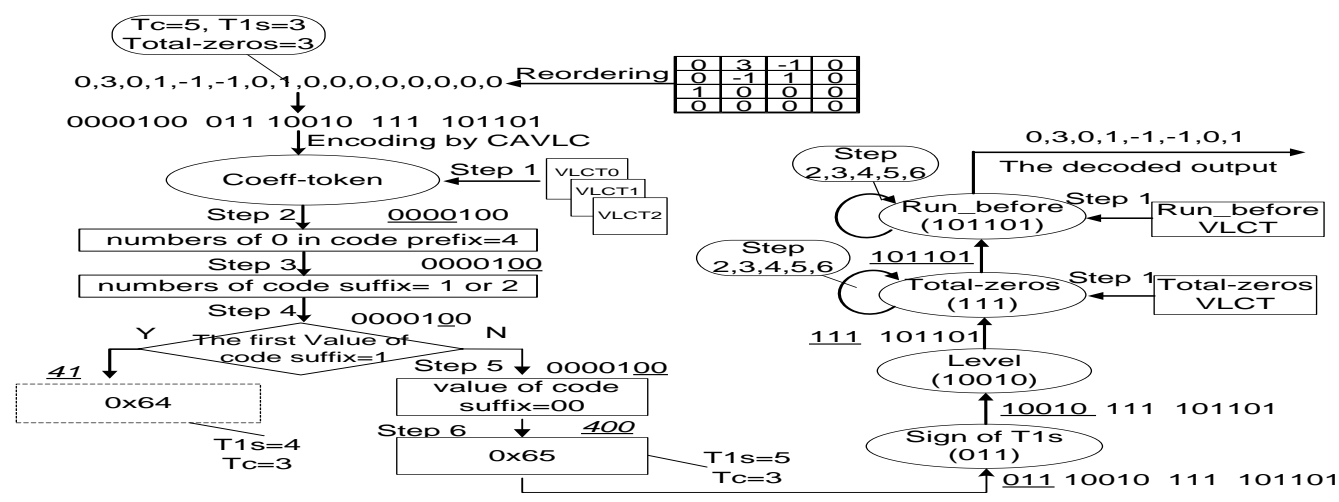

Figure 3. A Decoding Example with our Proposed Scheme

From Figure 3, we can clearly see the CAVLC decoding process of an example with our proposed scheme. The decoding process for our proposed scheme is shown as follows:

Step 1, variable length table 0 of Coeff_token is selected as Table entry because of NC value $(0 \leq \mathrm{NC}<2)$;

Step 2, read input decoding bit-stream and take the numbers of 0 in code prefix (4)calculated from input decoded bit-stream as index key of the first level(4);

Step 3, look up hash Table address (4) in Table 4 because of length of code suffix (4), and get the length of code suffix for 1 or 2 . 
Step 4, as there are two possible values (1 or 2 ) in length of code suffix, we can get the right length of code suffix (2) after judging the first value of code suffix (0) again.

Step 5, read code suffix (00) from the input decoded bit-stream according to length of code suffix(2) and take it as index key of the second level(00)

Step 6, through index key of the one and second level (400), we can find the decoded codeword (0x64) in table 4, at last, transform 0x64 into T1s=3, Tc=5.

After decoding the Coeff_token, level, sign of T1s, Total_zeros and Run_before syntax elements are decoded in sequence. As to Total_zeros and Run_before, the decoding steps are the same as the syntax elements of Coeff_token. At last, we could get the decoded output

\section{Simulation Results and Analysis}

In order to verify the effectiveness of our proposed method above, we take some experiments. In this section, our designed experiments mainly include two parts: experimental environment and reduce Table look-up time. In our experiments, we compared our proposed method compared with conventional TLSS, TLBS methods in the performance of Table lookup time.

\subsection{Simulation Environment}

The simulation environment was conducted on a Intel $2 \mathrm{GHz}$ processor, $1 \mathrm{~GB}$ memory capacity, Intel Windows XP operating system. Table 8 shows some parameters of test sequences, including the name, resolutions, frame rate and frame number of test sequences in our simulation experience. Some common encoding parameters are shown at Table 9.

Table 8. Parameters of Test Sequences

\begin{tabular}{|l|l|l|l|}
\hline Sequence(SEQ) & \multicolumn{1}{|c|}{ resolution } & Frame rate & \#frames \\
\hline Forman(FM) & CIF $(176 \times 144)$ & 25 & 30,90 \\
\hline Crew(C) & CIF $(176 \times 144)$ & 25 & 30,90 \\
\hline Paris(P) & CIF $(176 \times 144)$ & 25 & 30,90 \\
\hline Wal(W) & CIF $(176 \times 144)$ & 25 & 30,90 \\
\hline Football(FB) & QCIF $(352 \times 288)$ & 25 & 30,90 \\
\hline Harbour(H) & QCIF $(352 \times 288)$ & 25 & 30,90 \\
\hline Mobile(M) & QCIF $(352 \times 288)$ & 25 & 30,90 \\
\hline Soccer(S) & QCIF $(352 \times 288)$ & 25 & 30,90 \\
\hline
\end{tabular}

Table 9. Encoding Parameters

\begin{tabular}{|c|c|}
\hline Profile & Baseline \\
\hline SATD (Hadamard) & On \\
\hline RDOptimization & 1 \\
\hline RDO & On (fast algorithm) \\
\hline MV search range & \pm 32 pixels \\
\hline Reference frame & 5 frames \\
\hline QP & $24,28,32$ \\
\hline Motion search & Fast search \\
\hline Intra interval & 0 \\
\hline Motion search & Fast search \\
\hline SymbolMode & 0 (CAVLC is used) \\
\hline QPPrimeYZeroTransform BypassFlag & 0 (lossless) \\
\hline File & Vlc.c \\
\hline Encoder & JM 16.2 [12] \\
\hline
\end{tabular}




\subsection{Reduce Table Look-up Time}

In this subsection, we mainly evaluate the performance of Table look-up time with our proposed algorithm, which was compared with TLSS and TLBS methods in different sequences, different frames and QP value. The experimental results are shown in Table 10 as follows.

Table 10. The Comparison Results in Table Look Up Time for Three Methods

\begin{tabular}{|c|c|c|c|c|c|c|c|c|c|c|}
\hline$\backslash$ & QP & \multicolumn{3}{|c|}{24} & \multicolumn{3}{|c|}{28} & \multicolumn{3}{|c|}{32} \\
\hline $\begin{array}{c}\text { SE- } \\
\text { Q }\end{array}$ & $\begin{array}{l}\text { Fra- } \\
\text { mes }\end{array}$ & TLSS & TLBS & Ours & TLSS & TLBS & Ours & TLSS & TLBS & Ours \\
\hline \multirow{2}{*}{ FM } & $30 \mathrm{fs}$ & 91452424 & 703654562 & 64022671 & 67444596 & 54798689 & 4868503 & 46882240 & 36675651 & 33249835 \\
\hline & $90 \mathrm{fs}$ & 306339376 & 239356324 & 219563835 & 208683480 & 167782033 & 149423914 & 160228410 & 128135788 & 112836908 \\
\hline \multirow{2}{*}{$\mathrm{C}$} & $30 \mathrm{fs}$ & 106440956 & 72655340 & 76533631 & 87563480 & 69727275 & 63915094 & 66303640 & 53675677 & 47594250 \\
\hline & $90 \mathrm{fs}$ & 303817596 & 240763544 & 217477176 & 231906120 & 176903672 & 151872774 & 166929040 & 130367557 & 118132188 \\
\hline \multirow{2}{*}{$\mathrm{P}$} & $30 \mathrm{fs}$ & 67843250 & 53654235 & 48463850 & 56537290 & 52066743 & 50470973 & 49427280 & 37890456 & 34947884 \\
\hline & $90 \mathrm{fs}$ & 179095793 & 13445360 & 12485000 & 154580870 & 12867546 & 11120958 & 130896338 & 98677102 & 92201640 \\
\hline \multirow[b]{2}{*}{ W } & $30 \mathrm{fs}$ & 127718726 & 935691723 & 88219806 & 112815480 & 83016734 & 76294810 & 83498650 & 64667543 & 57197705 \\
\hline & $90 \mathrm{fs}$ & 361732130 & 27456978 & 254670549 & 311956514 & 245780426 & 218501055 & 251010610 & 19534780 & 174243486 \\
\hline \multirow{2}{*}{ FB } & $30 \mathrm{fs}$ & 121012110 & 90335461 & 86228407 & 10646260 & 84654087 & 7859623 & 74306817 & 6067833 & 5375746 \\
\hline & $90 \mathrm{fs}$ & 291077420 & 239347533 & 210998130 & 210894200 & 178905664 & 151461901 & 166631660 & 70257890 & 52469854 \\
\hline \multirow{2}{*}{$\mathrm{H}$} & $30 \mathrm{fs}$ & 110447540 & 88673652 & 81960188 & 81513646 & 65489076 & 59499007 & 42399550 & 3371096 & 3057059 \\
\hline & $90 \mathrm{fs}$ & 340546073 & 25808654 & 239962010 & 241007480 & 189056468 & 168122731 & 206009120 & 167441435 & 144991633 \\
\hline \multirow{2}{*}{ M } & $30 \mathrm{fs}$ & 125115200 & 91547043 & 86816152 & 85790423 & 65140457 & 58401646 & 37523680 & 29436734 & 26425126 \\
\hline & $90 \mathrm{fs}$ & 390783324 & 293654484 & 267386109 & 28507620 & 21975458 & 19900433 & 125915116 & 94698965 & 88743036 \\
\hline \multirow[t]{2}{*}{ S } & $30 \mathrm{fs}$ & 48479094 & 38635442 & 35457044 & 33094810 & 25673322 & 23996231 & 19407230 & 15265582 & 13728886 \\
\hline & $90 \mathrm{fs}$ & 161562740 & 135564536 & 113706161 & 110695448 & 84675530 & 78683115 & 67301960 & 53068420 & 48054257 \\
\hline
\end{tabular}

From Table 10, we could find that our proposed algorithm has superior results than other algorithms and shows about $40 \%$ saving time compared to the standard TLSS method. TLBS follows. The main reason for reducing Table look-up time for CAVLC decoding in H.264/AVC is that because our proposed algorithm uses a hash-index query method to lookup Table for CAVLC decoding, which could reduce lots of the operations of looking up and judging code length and code prefix and codeword by a hash-index Table built, therefore saving a lot of Table look-up time.

\section{Conclusion}

Table look-up is a very important operation for CAVLC decoding in H.264/AVC, which could seriously affect the efficiency of CAVLC decoding. In this paper, a quick a Table lookup optimization scheme based on hash-index query is proposed to solve the problem of long table look-up time for CAVLC decoding in H.264/AVC. Specifically, in our schemes, after finding the existing relationships between code length and numbers of 0 in code prefix, we uses hash Table and index technology to reduce Table look-up time. The simulation results show that our proposed schemes based on hash-index query can reduce about $40 \%$ Table look-up time for CAVLC decoding compared with TLSS method, without degrading video quality. 


\section{Acknowledgements}

The authors would like to thank the anonymous reviewers for their constructive opinions in improving this paper. The work was supported by the Joint Funds of the National Natural Science Foundation of China (No.U2012A002D01);The Strategic Emerging Industries Special of Guangdong Province (No.2012A09100013-2012BAF11B04-5150).Foundation for Distinguished Young Talents in Higher Education of Guangdong (No.LYM11057); Doctoral Project for Natural Science Foundation of Guangdong Province (No.S2012040006666).

\section{References}

[1] Joint Video Specification (ITU-T Rec. H.264|ISO/IEC 14496-10)-Joint Committee Draft Joint Video Team (JVT) of ISO/IEC MPEG and ITU-T VCEG, Doc. JVT-G050r1.doc, (2002).

[2] J. Lee, C. Park and S. Ha, "Memory Access Pattern Analysis and Stream Cache Design for Multimedia Applications", Proc. Asia and South Pacific, Design Automation Conf (DAC), Kitakyushu, Japan, (2003) January 21-24.

[3] H.-Y. Lin, Y.-H. Lu, B.-D. Liu and J.-F. Yang, "A highly Efficient VLSI architecture for H.264/AVC CAVLC Decoder, IEEE Transactions on multimedia, vol. 10, no. 1, (2008).

[4] B.-Y. Lee and K.-K. Ryoo, "A Design of High-Performance Pipelined Architecture for H.264/AVC CAVLC Decoder and Low-Power Implementation”, IEEE Transactions on Consumer Electronics, vol. 56, no. 4, (2010).

[5] K.-Y. Wang, B.-S. Kim, S.-S. Lee, D.-S. Kim and D.-J. Chung, "A novel low-cost high-throughput CAVLC decoder for H.264/AVC", IEICE Transactions on Information and Systems, E94-D, vol. 4, (2011).

[6] C.-H. Fang and C.-P. Fan, "Very-large-scale integration design of a low-power and cost-effective contextbased adaptive variable length coding decoder for H.264/AVC portable applications", IET Image Processing, vol. 6, no. 2, (2012).

[7] Y. H. Moon, G. Y. Kim and J. H. Kim, "An Efficient Decoding of CAVLC in H.264/AVC Video Coding Standard", IEEE Trans. on Consumer Electronics, vol. 51, no. 3, (2005).

[8] D. Lu, G. Liu and Z. Lingli, "An optimization for CAVLC code Table lookup algorithm in H. 264 decoder", 2011 2th International Symposium on Intelligence Information Processing and Trusted Computing, Hubei, China, (2011) October, pp. 79-82.

[9] J. Y. Lee, J. J. Lee and S. M. Park, "New Lookup Tables and Searching Algorithms for Fast H.264/AVC CAVLC Decoding", IEEE Trans Circuits and System Video Technology, vol. 20, no. 7, (2010).

[10] Y. H. Kim, Y. J. Yoo and J. Shin, "Memory-Efficient H.264/AVC CAVLC for Fast Decoding”, IEEE Trans On Consumer Electronics, vol. 52, no. 3, (2006).

[11] G. Sullivan and G. Bjontegaard, "Recommended simulation common conditions for H.26L coding efficiency experiments on low-resolution progressive-scan source material”, ITU-T VCEG, Doc. VCEG-N81, (2001).

[12] K. Suhring, "JM 16.2 software”, http://iphome.hhi.de/suehring/tml/.

\section{Authors}

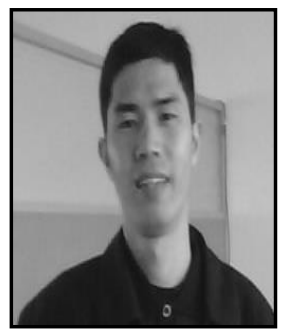

JianHua Wang, he was born on February 6, 1982 in Guangdong, China. He received his B.S degree in Electronic Information Science and Technology from Shaoguan University, Guangdong, China, in 2006. Currently he is pursuing Ph.D degree in Control Science and Engineering at Guangdong University of Technology. His research interests include $3 \mathrm{G}$ wireless video transmission, cyber-physical systems, IoT and wireless sensor networks. 


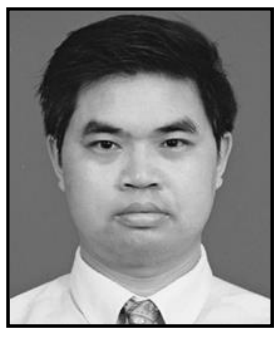

LiangLun Cheng, he was born on August 22, 1964 in Hubei. He received his M.S and Ph.D degrees from Huazhong University of Science and Technology, Hubei, China in 1992 and Chinese academy of Sciences Jilin, china in 1999 respectively. He is a Prof and doctoral supervisor of Guangdong University of Technology. His research interests include RFID and WSN, IoT and CPS, production equipment and automation of the production process, embedded system, the complex system modeling and its optimization control, software of automation and information, etc.,

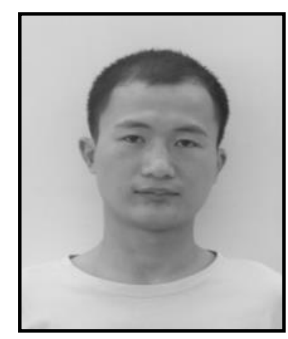

Jun Liu, he was born on October 11, 1986 in Hubei, China. He received his M.S degree in Control Science and Engineering from Guangdong University of Technology, Guangdong, China, in 2012. Currently he is pursuing Ph.D degree in Control Science and Engineering at Guangdong University of Technology. His research interests include 3G wireless video transmissions, cyber-physical systems and wireless sensor networks.

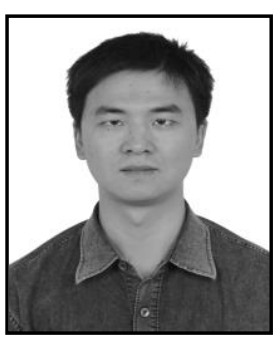

Tao Wang, he received the PHD Degree in network security from Sun Yat-Sen University, Guangzhou, China, in 2010. His current research interest includes context-aware computing, service composition, protocol optimization in wireless sensor network and cyber-physical system. 
International Journal of Signal Processing, Image Processing and Pattern Recognition Vol.7, No.3 (2014) 